\title{
PROPER HOLOMORPHIC MAPPINGS AND THE COWEN-DOUGLAS CLASS
}

\author{
STEVEN G. KRANTZ AND NORBERTO SALINAS
}

(Communicated by Palle E. T. Jorgensen)

\begin{abstract}
We study the relationship between intersection theory for analytic varieties and membership of $n$-tuples of Toeplitz operators in the CowenDouglas class $B_{m}(\Omega)$. Connections with holomorphic mappings are discussed.
\end{abstract}

\section{INTRODUCTION}

In this note we provide evidence that a close relationship exists between intersection theory and membership in the Cowen-Douglas class $B_{m}(\Omega)$ (see [6, 7]) of certain $n$-tuples $T_{\psi}$ of Toeplitz operators with bounded antiholomorphic symbols $\psi$, acting on the Bergman space over a pseudoregular bounded domain $\Delta \subseteq \mathbb{C}^{n}$. Here a pseudoregular domain is one on which the $\bar{\partial}$-Neumann operator $N$ is compact in the $L^{2}$ topology (see [17]). (Observe that Bell's Condition $\mathrm{R}$ is necessary for pseudoregularity; see [1].)

The above relationship stems from the fact that such $n$-tuples $T_{\psi}$ belong to the class $B_{m}(\Omega)$, where $\Omega$ is a bounded domain in $\mathbb{C}^{n}$ and $m \in \mathbb{Z}_{+}$, if and only if $\bar{\psi} \in H^{\infty}(\Delta)$ is an $m$-analytic cover of $\Omega$, i.e., $\Omega \subseteq \bar{\psi}(\Delta)$, and $\bar{\psi}: \Lambda=\bar{\psi}^{-1}(\Omega) \rightarrow \Omega$ is a proper mapping of multiplicity $m$. The proof of this result was given in [14]. In the course of this proof, an important property of proper holomorphic mappings was rediscovered (see the proof of $[14$, Theorem 3.5]):

Let $\phi: \Lambda \rightarrow \Omega$ be a proper holomorphic mapping of multiplicity $m$, so that $\operatorname{Card}\left(\phi^{-1}(\{w\})\right)=m \forall w \notin \phi(\mathscr{X})$, where $\mathscr{X}=\left\{z \in \Lambda: \operatorname{det} \phi^{\prime}(z)=0\right\}$ is the critical manifold of $\phi$. For each $z \in \Lambda$ let $\mathscr{O}(z)$ be the algebra of germs of holomorphic functions in neighborhoods of $z$ and $\mathscr{F}_{\phi}(z)$ be the ideal in

Received by the editors April 9, 1991 and, in revised form, May 9, 1991.

1991 Mathematics Subject Classification. Primary 47B35, 32H35.

Key words and phrases. Cowen-Douglas class, Toeplitz operator, intersection theory, multiplicity, pseudoregular domain.

Both authors were supported in part by the National Science Foundation. We have benefited from several useful conversations with Daowei Ma. His ideas contributed decisively to the final form of this paper. 
$\mathscr{O}(z)$ generated by the components of $\phi-\phi(z)$. Then

$$
m(w)=\sum_{\phi(z)=w} \operatorname{dim}\left[\mathscr{O}(z) / \mathscr{I}_{\phi}(z)\right], \quad w \in \Omega,
$$

is constant on $\Omega$ and hence coincides with $m$.

Property $(*)$ for proper holomorphic mappings (at least for compact complex manifolds) is well understood among algebraic geometers and is a generalization of a theorem of Remmert and Stein (see [16, Theorem 15.1.9]); a treatment of the result may be found in [10, Corollary 3.13, Proposition 3.20] and [5, $\S \S 10.2$, 12.6]. We shall prove it here and provide a flow chart of appropriate references. We shall also present a simplification of [14, Theorem 3.5]. We point out that in [14], property $(*)$ is proved using operator theoretic techniques. We believe that the arguments provided here are more natural.

Actually, condition $(*)$ is essentially equivalent to another interesting property of proper holomorphic mappings brought to light by the above-mentioned connection between the Cowen-Douglas class and intersection theory. To put the hypotheses of our theorem in perspective, we note that the papers $[13,20]$ provide examples of pseudoconvex domains on which the $\bar{\partial}$-Neumann operator $N$ is noncompact in the $L^{2}$ topology.

Theorem 1.1. Let $U$ be a smoothly bounded, pseudoregular domain in $\mathbb{C}^{n}$, i.e., a pseudoconvex domain on which the $\bar{\partial}$-Neumann operator $N$ is compact in the $L^{2}$ topology. Also, let $H^{2}(U)$ be the Bergman space on $U$, i.e., the subspace of $L^{2}(U)$ of holomorphic functions on $U$. Given a bounded holomorphic mapping $\phi: U \rightarrow \mathbb{C}^{n}$ whose components are in $C^{1}(\bar{U})$, let $T_{\phi}$ be the n-tuple of multiplication operators by the components of $\phi$ acting on $H^{2}(U)$. For a given domain $\Omega \subseteq \mathbb{C}^{n}$, the following conditions are equivalent:

(1) $\Omega \subseteq \phi(U)$ and $\phi: \Lambda \equiv \phi^{-1}(\Omega) \rightarrow \Omega$ is a proper mapping of multiplicity $m$.

(2) For each $w \in \Omega$, the linear space $\operatorname{Ran}\left(T_{\phi-w}\right)$ defined by

$$
\operatorname{Ran}\left(T_{\phi-w}\right)=\left\{\sum_{j=1}^{n}\left(\phi_{j}-w_{j}\right) f_{j}: f_{j} \in H^{2}(U), 1 \leq j \leq n\right\}
$$

is closed in $H^{2}$ and has codimension $m$.

(3) For each $w \in \Omega$ the linear space $\operatorname{Ran}\left(T_{\phi-w}\right)$ is closed and the map $w \rightarrow E_{\phi}(w)=H^{2}(U) / \operatorname{Ran}\left(T_{\phi-w}\right)$ defines an m-dimensional holomorphic vector bundle $E_{\phi}$ that gives a natural holomorphic vector bundle structure to the assignment

$$
\Omega \ni w \mapsto \bigoplus_{\phi(z)=w} \mathscr{O}(z) / \mathscr{I}_{\phi}(z)
$$

Corollary 1.2. Let $U$ and $\Omega$ be two (smoothly bounded) pseudoconvex domains in $\mathbb{C}^{n}$, and assume that $U$ is pseudoregular. Also, let $\phi: U \rightarrow \Omega$ be a proper holomorphic mapping of multiplicity $m$. Then the map $w \rightarrow E_{\phi}(w), w \in$ $\Omega$, defines an m-dimensional holomorphic vector bundle over $\Omega$.

Remark 1.3. (a) There are several important examples of pseudoregular domains. For example, domains of finite type are pseudoregular. Examples of 
domains that are not of finite type in general, but that are still pseudoregular, are domains satisfying Catlin's property $P$ (see [4]). Typically, pseudoconvex Reinhardt domains with no analytic disks in their boundaries are pseudoregular (see [21, 19]). On the other hand, the new work of Boas and Straube [3] shows that smooth convex domains satisfy Condition $\mathrm{R}$ and hence are pseudoregular.

(b) It is natural to ask the following question: with $\phi: U \rightarrow \Omega$ as in the above corollary, what is the behavior of $E_{\phi}(w)$ as $w$ approaches some point in $\partial \Omega$ ? As pointed out previously, $U$ satisfies Bell's Condition $R$, i.e., the Bergman projection $P: L^{2}(U) \rightarrow H^{2}(U)$ leaves $C^{\infty}(\bar{U})$ invariant. It follows that $\phi$ can be extended smoothly to $\partial U$ (see [2] or [8]). (Note that $\operatorname{Ran}\left(T_{\phi-w}\right.$ ) is no longer closed when $w \in \partial U$. In fact, we suspect that, in most cases, $\operatorname{Ran}\left(T_{\phi-w}\right)$ is dense in $H^{2}(U)$ when $w \in \partial U$.) In case the boundaries of $U$ and $\Omega$ are real analytic and pseudoconvex, then $\phi$ continues holomorphically past the boundary (see [9]). Formally, the vector bundle $E_{\phi}$ seems to depend on the Hilbert space $H^{2}(U)$, but that turns out not to be the case. Here is why.

Let $\phi: \Lambda \rightarrow \Omega$ be a proper map. Consider the direct image sheaf $\phi_{*} \mathscr{O}_{\Lambda}$. The fibre at a point $y \in \Omega$ is

$$
\begin{array}{r}
\left(\phi_{*} \mathscr{O}_{\Lambda}\right)_{y} \otimes_{\mathscr{O}_{\Omega, y}}\left(\mathscr{O}_{\Omega, y} / m\right)=\bigoplus_{x \in \phi^{-1}(y)}\left(\mathscr{O}_{\Lambda, x} \otimes_{\mathscr{O}_{\Omega, y}}\left(\mathscr{O}_{\Omega, y} / m_{y}\right)\right) \\
=\bigoplus_{x \in \phi^{-1}(y)}\left(\mathscr{O}_{\Lambda, x} / \mathscr{O}_{\Lambda, x} m_{y}\right)=\bigoplus_{x \in f^{-1}(y)}\left(\mathscr{O}_{\Lambda, x} / I(\phi-\phi(x))\right) .
\end{array}
$$

By Criterion 2 in $[11$, p. 91$]$, the sheaf $\phi_{*} \mathscr{O}_{\Lambda}$ is locally free. That is, it is the sheaf of germs of a holomorphic vector bundle. This gives the assignment

$$
y \rightarrow \bigoplus_{x \in \phi^{-1} y} \mathscr{O}_{\Lambda, x} / I(\phi-\phi(x))
$$

a natural structure of holomorphic bundles that is independent of operatortheoretic considerations. Therefore, when $\phi$ continues holomorphically past the boundary, the vector bundle (whose associated sheaf is $\phi_{*} \mathscr{O}_{\Lambda}$ ) continues holomorphically past the boundary.

Thus we think of $\phi$ as a proper mapping of a larger domain that properly contains $\bar{U}$. As a result, we see that $w$ tending to $\partial U$ is no different from $w$ lying in the interior of $U$ and the statement of (b) holds even when $w$ lies in $\partial U$.

However, we must stress that the continuation of $E_{\phi}$ that we have specified is as a holomorphic vector bundle. The utility of $E_{\phi}$ in Theorem 1.1 is as a Hermitian vector bundle (see [16]). Thus, at this writing, we do not fully understand the boundary behavior of $E_{\phi}$.

\section{INTERSECTION NUMBERS AND MULTIPLICITY}

Definition 2.1. Let $f_{j} \in \mathscr{O}(0)$ be such that $f_{j}(0)=0,1 \leq j \leq n$, and assume that $\{0\}=\bigcap_{j=1}^{n} V_{j}$, where $V_{j}$ is the divisor associated with $f_{j}, 1 \leq j \leq n$. 
Given $g \in \mathscr{O}(0)$, the residue of the meromorphic form

$$
\alpha=\frac{g(z) d z_{1} \wedge d z_{2} \wedge \cdots \wedge d z_{n}}{\prod_{j=1}^{n} f_{j}(z)}
$$

is defined for a sufficiently small $\varepsilon>0$ by

$$
\operatorname{Res}\left(\alpha, L^{0}\right)=\int_{\Gamma_{\varepsilon}} \alpha,
$$

where $\Gamma_{\varepsilon}$ is the $n$-cycle $\left\{z \in \mathbf{C}^{n}:\left|f_{j}(z)\right|=\varepsilon, 1 \leq j \leq n\right\}$ oriented positively in the standard fashion (see [12, p. 649]).

Let $\beta(z)$ be the Bochner-Martinelli kernel, i.e., $\beta(z)=\eta(z) \wedge \omega(z)$, where

$$
\omega(z)=d z_{1} \wedge \cdots \wedge d z_{n}
$$

and

$$
\eta(z)=\sum_{j=1}^{n}(-1)^{j-1} \frac{\bar{z}_{j}}{[z]^{2 n}} d \bar{z}_{1} \wedge \cdots \wedge d \bar{z}_{j-1} \wedge d \bar{z}_{j+1} \wedge \cdots \wedge d \bar{z}_{n} .
$$

Also, let $\gamma$ be the Cauchy kernel, i.e.,

$$
\gamma(z)=\frac{\omega(z)}{\prod_{j=1}^{n} z_{j}} .
$$

Lemma 2.2 (The Degree Lemma). Let $F=\left(f_{1}, \ldots, f_{n}\right)$ be such that $f_{j} \in$ $\mathscr{O}(0)$ and zero is an isolated zero of $F$. Let $B_{\varepsilon}$ be the ball with center 0 and radius $\varepsilon$. Then, for $\varepsilon$ sufficiently small, we have

$$
(2 \pi i)^{-n} \operatorname{Res}\left(F^{*} \gamma, 0\right)=\frac{C_{n}}{(2 \pi i)^{n}} \int_{\partial B_{\varepsilon}} F^{*} \beta=\operatorname{deg}(F, 0),
$$

where $\operatorname{deg}(F, 0)$ denotes the degree of $F$ at zero and $C_{n}>0$ depends only on $n$.

Proof. If $F$ is nondegenerate then this is little more than a change of variables.

The degenerate case requires an extra argument. It uses the ideas of [GH, pp. 651, 666]. We shall not provide the details here.

Lemma 2.3 (The Residue Lemma). Let $\phi: \Lambda \rightarrow \Omega$ be as in Theorem 1.1. Further, let $B_{\varepsilon}$ be a ball in $\mathbb{C}^{n}$ such that $\overline{B_{\varepsilon}} \subseteq \Omega$ and $\Lambda^{\prime} \subset \subset \Lambda$ be a smoothly bounded subdomain of $\Lambda$ such that $\phi^{-1}\left(B_{\varepsilon}\right) \subseteq \Lambda^{\prime}$. Then, for every $w \in B_{\varepsilon}$, we have

$$
(2 \pi i)^{n} \sum_{\phi(z)=w} \operatorname{Res}\left(\phi^{*} \gamma(\cdot-w), z\right)=C_{n} \int_{\partial \Lambda^{\prime}} \phi^{*} \beta(\cdot-w) .
$$

Proof. This is an exercise with Stokes's theorem. See [12, p. 656].

Lemma 2.4 (The Intersection Number Lemma). Let $\phi: \Lambda \rightarrow \Omega$ be as in Theorem 1.1. Then, for every $z \in U$,

$$
\operatorname{deg}(\phi-\phi(z), z)=\operatorname{dim}_{\mathbb{C}}\left[\mathscr{O}(z) / \mathscr{I}_{\phi}(z)\right] .
$$

Proof. There is an elegant discussion of this result in [12, pp. 669-670]. The formula comes from relating the topological definition of intersection number to the algebraic definition. 
Theorem 2.5 (The Multiplicity Theorem). Let $\phi: \Lambda \rightarrow \Omega$ be as in Theorem 1.1, and let. $B_{\varepsilon}$ be as in Lemma 2.4. Then for every $w \in B_{\varepsilon}$, we have

$$
m(w)=\frac{C_{n}}{(2 \pi i)^{n}} \int_{\partial \Lambda^{\prime}} \phi^{*} \beta(\cdot-w) .
$$

In particular, $m(\cdot)$ is a continuous function on $\Omega$ and hence coincides with the multiplicity $m$ of $\phi$. (Thus, in particular, property (*) from $\S 1$ holds.)

Proof. This follows from Lemmas 2.2, 2.3, and 2.4.

\section{The Cowen-Douglas class}

For $\Omega \subseteq \mathbb{C}$ a connected open set, the Cowen-Douglas class $B_{m}(\Omega)$ is defined as follows: Let $\mathscr{H}$ be a Hilbert space and $\mathscr{L}(\mathscr{H})$ be the bounded linear operators on $\mathscr{H}$. An element $T \in \mathscr{L}(\mathscr{H})$ lies in $B_{m}(\Omega)$ if

(1) $\Omega \subseteq \sigma(T)$;

(2) $(T-\omega) \mathscr{H}=\mathscr{H} \quad \forall \omega \in \Omega$;

(3) $\bigvee_{\omega \in \Omega} \operatorname{ker}(T-\omega)=\mathscr{H}$;

(4) $\operatorname{dim} \operatorname{ker}(T-\omega)=n \quad \forall \omega \in \Omega$.

Here $\sigma(T)$ is the spectrum of $T$.

Let $\Omega$ be a bounded domain in $\mathbb{C}^{n}$. Any of the equivalent conditions given in the next proposition for an $n$-tuple $T$ satisfying the hypotheses will guarantee that $T^{*}$ is in the Cowen-Douglas class $B_{m}(\Omega)$ (see [6;18, Theorem 3.4]). As we shall see below, such hypotheses are automatically satisfied in our situation.

Proposition 3.1. Let $\mathscr{H}$ be a (separable, infinite-dimensional, complex) Hilbert space and $T$ be a commuting $n$-tuple of operators on $\mathscr{H}$ such that

$$
\operatorname{span}_{w \in \Omega} \operatorname{Ker}\left[(T-w)^{*}\right]
$$

is dense in $\mathscr{H}$. Then the following conditions are equivalent:

(a) The linear space $\operatorname{Ran}(T-w)$ is closed and has codimension $m$ for every $w \in \Omega$.

(b) Zero is an isolated point of $\sigma\left(\left|(T-w)^{*}\right|\right)$ for every $w \in \Omega$, and the spectral projection $P(w)$ corresponding to $\{0\}$ is a continuous function of $w$, with $\operatorname{Rank}[P(w)]=m$ for every $w \in \Omega$. Here we have used the notation $|S|=\left(\sum_{j=1}^{n} S_{j}^{*} S_{j}\right)^{1 / 2}$.

(c) In addition to property (b) above, we have $w \rightarrow P(w), w \in \Omega$, defines an antiholomorphic vector bundle over $\Omega$.

Proof. See [7, Theorem 2.2; 18, Theorem 3.4].

Theorem 3.2. Let $U$ be a smoothly bounded pseudoregular domain and $\phi: U \rightarrow$ $\mathbb{C}^{n}$ be a holomorphic mapping that extends continuously to $\partial U$. Also, assume that $\Omega$ is another bounded domain such that $\Omega \subseteq \phi(U)$. Then the following conditions are equivalent:

(1) $T_{\phi}^{*}$ is in $B_{m}(\Omega)$.

(2) $\phi: \Lambda=\phi^{-1}(\Omega) \rightarrow \Omega$ is a proper mapping of multiplicity $m$.

Proof. For $(\mathrm{a}) \Rightarrow(\mathrm{b})$, let $\left\{z_{k}\right\}$ be a sequence in $\Lambda$ such that $z_{k} \rightarrow z_{0} \in \partial \Lambda$. Assume, seeking a contradiction, that $\phi\left(z_{k}\right) \rightarrow w_{0} \in \Omega$. Since $\Omega \cap \sigma_{r e}\left(T_{\phi}\right)=\varnothing$, because $T_{\phi}^{*} \in B_{m}(\Omega)$, we see that $T_{\phi-w_{0}}$ is essentially right invertible; so by [14, 
Proposition 2.2], $\phi-w_{0}$ is bounded away from zero near $\partial U$. This means that $\left\{z_{k}\right\}$ cannot have any limit point in $\partial U$, and hence $z_{0} \in U$. By continuity of $\phi$ on $U, w_{0}=\phi\left(z_{0}\right)$. Therefore, $z_{0} \in \phi^{-1}\left(\left\{w_{0}\right\}\right) \subseteq \Lambda$, which contradicts $z_{0} \in \partial \Lambda$. It follows that $\phi$ is proper. Now, let $w \in \Omega$ be a fixed noncritical value of $\phi$, i.e., $w \notin \phi(\mathscr{X})$, where $\mathscr{Z}$ is the zero set of the Jacobian of $\phi$. Let $X=\phi^{-1}(\{w\})$ and $k(\cdot, \cdot)$ be the Szegö kernel of $U$, i.e., the reproducing kernel of $H^{2}(U)$. Since $k(\cdot, z) \in \operatorname{Ker}\left[\left(T_{\phi-w}\right)^{*}\right]$ for every $z \in X$, we see that

$$
\operatorname{deg}(\phi)=\operatorname{Card}(X) \leq \operatorname{dim}\left[\operatorname{Ran}\left(T_{\phi-w}\right)^{\perp}\right]=m .
$$

The opposite inequality follows as in the proof of [14, Theorem 3.5].

For the proof that (b) $\Rightarrow(\mathbf{a})$, assume that $\phi$ is an $m$-analytic cover of $\Omega$. By [17, Theorem 2.3] it follows that $\sigma\left(T_{Z}\right)=\bar{U}$ and $\sigma_{e}\left(T_{Z}\right)=\partial U$. Since $T_{Z}$ is essentially normal (because $U$ is assumed to be pseudoregular, see again [17, Theorem 2.3]), $T_{\phi}$ is also essentially normal, and hence

$$
\phi(\partial U)=\sigma_{e}\left(T_{\phi}\right)=\sigma_{r e}\left(T_{\phi}\right) .
$$

Since $\Omega \subseteq \phi(U)$, we deduce that $T_{\phi-w}$ is essentially right invertible for every $w \in \Omega$. This means that $\operatorname{Ran}\left(T_{\phi-w}\right)$ is closed and

$$
p(w)=\operatorname{dim}\left[\operatorname{Ran}\left(T_{\phi-w}\right)^{\perp}\right]<\infty,
$$

for every $w \in \Omega$. Let $w \in \Omega \backslash \phi(\mathscr{X})$. Then an argument similar to one given above (using the Bergman kernel) yields

$$
m=\operatorname{Card}\left(\phi^{-1}(\{w\})\right) \leq p(w) .
$$

The opposite inequality is proved employing an argument used in the proof of (b) $\Rightarrow$ (a) of [14, Theorem 3.5]. By Proposition 3.1, $T_{\phi}^{*} \in B_{m}(\Omega)$.

Proof of Theorem 1.1. (a) $\Rightarrow$ (b) follows from Theorem 3.2.

(b) $\Rightarrow$ (c) follows from Proposition 3.1, except for the last assertion (i.e., the fibre isomorphism statement). The proof of that fact uses an argument identical to one given in the proof of [14, Theorem 3.5].

In order to proof that $(\mathrm{c}) \Rightarrow(\mathrm{a})$, note that $\sigma\left(T_{\phi}\right)=\overline{\phi(U)}$ because we are assuming that $\phi \in C^{1}(\bar{U})$ (see $[15, \S 4]$ ). Since $\Omega \cap \sigma_{r e}\left(T_{\phi}\right)=\varnothing$, because $T_{\phi}^{*} \in B_{m}(\Omega)$ (so that $T_{\phi-w}$ is right invertible for every $w \in \Omega$ ), and $\partial \phi(U) \subseteq$ $\sigma_{r e}\left(T_{\phi}\right)$ by [14, Proposition 2.2], we deduce that $\Omega \subseteq \phi(U)$. Now the desired conclusion follows from Theorem 3.2.

\section{REFERENCES}

1. S. Bell, Biholomorphic mappings and the $\bar{\partial}$-problem, Ann. of Math. (2) 114 (1981), 103-113.

2. S. Bell and D. Catlin, Boundary regularity of proper holomorphic mappings, Duke Math. J. 49 (1982), 385-396.

3. H. Boas and E. Straube, Sobolev estimates for the $\bar{\partial}$-Neumann operators on domains in $\mathbf{C}^{n}$, preprint, 1990.

4. D. Catlin, Global regularity of the $\bar{\partial}$-Neumann problem, Proc. Sympos. Pure Math., vol. 41 , Amer. Math. Soc., Providence, RI, 1984.

5. E. M. Chirka, Complex analytic sets, Kluwer, Dordrecht, 1989.

6. M. Cowen and R. Douglas, Operators possessing an open set of eigenvalues, Colloq. Math. Budapest 35 (1981), 323-341. 
7. R. Curto and N. Salinas, Generalized Bergman kernels and the Cowen-Douglas theory, Amer. J. Math. 106 (1984), 447-488.

8. K. Diederich and J. Fornaess, Boundary regularity of proper holomorphic mappings, Invent. Math. 67 (1982), 363-384.

9. Math. Ann. 282 (1988), 681-700.

10. G. Fisher, Complex analytic geometry, Lecture Notes in Math., vol. 538, Springer-Verlag, Berlin, 1976.

11. H. Grauert and R. Remmert, Coherent analytic sheaves, Springer-Verlag, Berlin, 1985.

12. P. Griffiths and J. Harris, Principles of algebraic geometry, Wiley, New York, 1978.

13. S. Krantz, On the compactness of the $\bar{\partial}$-Neumann operator, Proc. Amer. Math. Soc. 103 (1988), 1136-1138.

14. Q. Lin and N. Salinas, Proper holomorphic maps and analytic Toeplitz N-tuples, Indiana Univ. Math. J. 39 (1990), 546-562.

15. M. Putinar, Spectral theory and sheaf theory. IV, Proc. Sympos. Pure Math., vol. 51, Amer. Math. Soc., Providence, RI, 1990.

16. W. Rudin, Function theory on the unit ball of $\mathbb{C}^{n}$, Springer-Verlag, New York, 1980.

17. N. Salinas, The $\bar{\partial}$-formalism and the $C^{*}$-algebra of the Bergman n-tuple, J. Operator Theory 22 (1989), 325-343.

18. _ The Grassmann manifold of a $C^{*}$-algebra and Hermitian holomorphic bundles, Operator Theory: Adv. Appl. 28 (1988), 267-289.

19. - Toeplitz operators and weighted Wiener-Hopf operators, pseudoconvex Reinhardt and tube domains, Trans. Amer. Math. Soc. (to appear).

20. Won-compactness of the $\bar{\partial}$-Neumann problem and Toeplitz $C^{*}$-algebras, Proc. Sympos. Pure Math., vol. 52, no. 3, Amer. Math. Soc., Providence, RI, 1991, pp. 329-334.

21. N. Salinas, A. Sheu, and H. Upmeier, Toeplitz operators on pseudoconvex domains and foliation $C^{*}$-algebras, Ann. of Math. (2) 130 (1989), 531-565.

Department of Mathematics, Washington University, St. Louis, Missouri 63130-4899

Department of Mathematics, University of Kansas, Lawrence, Kansas 66045-2142 